\title{
La transplantation hépatique avec donneur vivant parental: réflexion éthique à la lumière de notre expérience des trente premiers patients
}

\author{
Jean Bernard Otte
}

La majorité des candidats à la transplantation hépatique pédiatrique sont des enfants en bas âge : $65 \%$ étaient âgés de moins de 3 ans au moment de la greffe dans notre série de 400 enfants ; leur nombre excède, dans tous les pays, le nombre de petits donneurs pédiatriques. Il en a résulté une mortalité élevée en liste d'attente, qui fut de 16,5\% pour les 249 enfants inscrits dans notre centre de 1983 à 1990. Le développement d'alternatives au foie entier (foie réduit [1] et foie partagé [2] rend possible la greffe chez l'enfant d'une partie d'un foie d'adulte et a permis de réduire la mortalité préalable à la greffe à $10 \%$ des 70 enfants inscrits électivement en 1991-1992. Depuis lors, le nombre de donneurs a tendance à se stabiliser (voire à diminuer) alors que la demande s'est considérablement accrue, surtout chez l'adulte, en raison précisément du succès de la transplantation hépatique. Le résultat en a été une diminution de l'accès aux greffons adultes et une augmentation à nouveau de la mortalité avant la greffe (17,5\% des 40 enfants inscrits électivement en 1993). En outre, la durée de l'attente avant la greffe entraîne une dégradation de l'état de santé des candidats avant qu'un greffon soit enfin disponible. Les soins postopératoires en sont alourdis, ainsi que les coûts de la transplantation, avec dégradation des résultats, sans oublier l'anxiété et la souffrance liées à ces situations. Ces faits nous ont conduits à développer dans notre Institution un programme de transplantation hépatique pédiatrique avec donneur vivant parental. A la lumière de l'expérience de nos trente premiers patients, nous livrons une réflexion sur les principes éthiques qui guident cette activité et sur leur modulation telle qu'elle a résulté de la pratique.

\section{Protocole}

Un protocole de transplantation hépatique avec donneur vivant parental fut développé et ses aspects éthiques particuliers (équilibre des risques et des avantages pour donneur et receveur, autodétermination du donneur et justice) furent analysés en collaboration avec le centre d'Études Bioéthiques de l'Université Catholique de Louvain. Nous nous sommes inspirés de l'expérience développée précédemment à l'Université de Chicago [3]. Pour éviter toute forme de pression, notre option initiale fut de ne considérer que les demandes exprimées spontanément par les parents ou les grands-parents. Notre protocole fut approuvé en juillet 1992 par la Commission
d'Éthique Bio-Médicale Hospitalofacultaire de l'UCL et, en octobre 1992, par le Conseil d'Administration des Cliniques Saint-Luc. Nous avons cependant attendu une année avant de commencer l'application clinique pour nous assurer de sa nécessité et de l'intime conviction de tous les membres de l'équipe pluridisciplinaire concernant le bien-fondé de sa mise en œuvre. Le premier patient fut opéré le 28 juillet 1993.

- Sélection des candidats donneurs et receveurs

La sélection des donneurs comprend : (1) un examen médical réalisé par un interniste n'appartenant pas à l'équipe pluridisciplinaire de transplantation, chargé d'exclure tout facteur de risque médical ; cet examen vérifie la normalité de la fonction hépatique; (2) une évaluation psychologique par un psychiatre pour détecter les personnes à risque de décompensation psychique et s'assurer de la motivation et du choix libre de toute contrainte extérieure; (3) des examens radiographiques pour vérifier la possibilité du prélèvement; (4) le recueil du consentement éclairé du donneur en deux étapes : avant le début de l'évaluation et avant le prélèvement. 
De 1993 à 1995, 69 donneurs vivants potentiels ont été évalués : 36 mères, 31 pères, une grand-mère et un oncle. Quarante-quatre candidats ont été retenus dont 27 mères, 16 pères et une grand-mère. Trente donneurs ont subi le prélèvement (17 mères, 12 pères et une grand-mère) et dix ne l'ont pas subi (dans neuf cas parce que leur première option était celle du donneur cadavérique - leurs enfants ont été transplantés dans l'intervalle - et, dans un cas, parce que l'enfant est décédé avant la transplantation). L'indication de greffe a été reportée dans quatre cas.

La candidature de vingt-cinq donneurs n'a pas été retenue pour contre-indication médicale chez 14 , pour contre-indication chirurgicale chez 8 (anatomie vasculaire défavorable) et par choix intrafamilial dans 3 cas où la candidature simultanée des deux parents - d'une grand-mère et d'un oncle dans un cas particulier - pouvait être acceptée.

L'indication de greffe de foie étant posée, la décision de réaliser la greffe avec un donneur vivant tient compte des chances de succès de la transplantation qui doivent être supérieures aux risques d'échec afin de valoriser le plus possible la démarche du donneur. A cet effet, le protocole initial prévoyait de ne prendre en considération que les enfants souffrant d'une affection hépatique chronique en condition stable pour permettre leur transplantation élective. Trois exceptions (hépatite fulminante) ont été acceptées en cours d'expérience, ce qui nous a conduits récemment à remanier notre protocole, nous y reviendrons.

\section{Résultats de notre expérience des 30 premiers patients opérés entre août 1993 et octobre 1995}

\section{Donneurs}

L'âge médian des parents était de 31 ans (extrêmes : 19-42). Une grandmère âgée de 54 ans fut acceptée en raison de contre-indications médicales chez les parents (incompatibilité $\mathrm{ABO}$ chez le père et sclérose en
Dans 27 cas, nous avons réalisé une lobectomie hépatique gauche emportant les segments II et III de Couinaud; dans les trois autres cas, nous avons étendu l'hépatectomie à la plus grande partie du segment IV (sans toutefois prélever la veine sushépatique moyenne). Trois donneurs ne requirent aucune transfusion. Parmi les vingt-sept autres donneurs, dix-sept furent transfusés uniquement pendant le prélèvement, huit furent transfusés pendant et après le prélèvement et deux uniquement après le prélèvement. Un seul donneur reçut une unité de sang homologue ; tous les autres reçurent exclusivement du sang autologue (prélevé avant l'opération ou récupéré dans le champ opératoire).

La durée moyenne du séjour postopératoire fut de 5,3 jours (extrêmes : 4-7 jours); plusieurs donneurs ont quitté l'hôpital dès le $4^{\mathrm{e}}$ jour postopératoire. Une poussée modérée de cytolyse fut observée en postopératoire (pic moyen d'ALAT : 282 UI/1 extrêmes : 75-954 (valeurs normales : 4-32)). Le pic moyen de la bilirubine sérique postopératoire fut de $1,9 \mathrm{mg} / \mathrm{dl}$ (32 mM par dl) avec des extrêmes de 0,8 à $5,2 \mathrm{mg} / \mathrm{dl}$. Tous les donneurs avaient récupéré des tests hépatiques normaux 6 mois après l'opération.

Six patients ont développé une complication postopératoire : une discrète fuite biliaire à partir de la tranche de section hépatique qui guérit spontanément en quelques jours, trois hernies incisionnelles qui durent être opérées ultérieurement et deux cas d'épanchement pleural droit infraclinique. Tous les donneurs s'estimèrent complètement rétablis sur le plan physique dans les trois semaines suivant le prélèvement. La régénération hépatique mesurée par tomodensitométrie chez 23 donneurs était de $87 \%$ (valeur médiane) 6 mois après le prélèvement (extrêmes : 59 \%-134\%).

\section{Receveurs}

\section{- Indications}

Les indications de la greffe furent représentatives des affections hépatiques conduisant à la transplantation dans l'enfance : 23 atrésies biliaires après échec de la porto-entérostomie selon Kasaï [4], un syndrome d'Ala- gille, un déficit en $\alpha$-1-antitrypsine avec syndrome hépatopulmonaire sévère (shunt droit-gauche calculé de $43 \%$ ) [5], une cirrhose cryptogénétique, une cholestase familiale, deux hépatites fulminantes et une hépatite subfulminante.

\section{- Âge}

L'âge médian des receveurs au moment de la transplantation était de 19 mois (extrêmes : 4-94) ; onze enfants étaient âgés de moins d'un an. Leur poids médian était de $10 \mathrm{~kg}$ (extrêmes : 4,3-23) ; 17 enfants pesaient moins de $10 \mathrm{~kg}$. Le rapport médian entre le poids du greffon et le poids corporel du receveur était de 2,45\% (extrêmes : 0,91\%-6\%). Tous les enfants reçurent un greffon identique ou compatible dans le système $\mathrm{ABO}$ et avaient un cross-match négatif. Le temps d'attente médian entre la première admission des 27 enfants souffrant d'hépatopathie chronique et leur transplantation fut de 31 jours (extrêmes : 15-255). Dix-sept enfants étaient en condition stable et en attente à la maison ; les dix autres restèrent hospitalisés pour traitement médical de l'insuffisance hépatique. Les greffons hépatiques furent transplantés de façon orthotopique selon la technique standardisée du foie partiel gauche [6-8].

Nous avons observé un cas de thrombose artérielle et de thrombose portale successives du même greffon (corrigées par reconstruction chirurgicale) et six sténoses de l'anastomose biliaire qui furent également réopérées avec succès.

\section{- Immunosuppression}

L'immunosuppression fut induite par triple thérapie avec azathioprine, stéroïdes et ciclosporine A (17 patients) ou tacrolimus (13 patients). L'incidence de rejet aigu fut de $82 \%$ dans le groupe ciclosporine et de $53 \%$ dans le groupe Tacrolimus. Deux tiers des rejets aigus survenus sous ciclosporine s'avérèrent résistants aux stéroïdes et imposèrent de substituer le tacrolimus à la ciclosporine (un greffon fut perdu par rejet chronique et fut remplacé par un greffon cadavérique). Aucun rejet résistant aux stéroïdes ne survint sous tacrolimus mais cinq enfants développèrent un syn- 
drome lymphoprolifératif (E. Sokal, communication personnelle) lors d'une primo-infection par le virus EBV, de 6 à 9 mois après la greffe. Un enfant en décéda et un autre dût être retransplanté avec un greffon cadavérique pour rejet chronique développé après arrêt temporaire de l'immunosuppression.

Vingt-huit patients $(93,3 \%)$ sont en vie de 14 à 41 mois après la greffe (y compris les deux enfants retransplantés avec un greffon cadavérique). Nous avons déploré le décès d'un patient survenu un mois et demi après transplantation par récidive d'une hépatite subfulminante d'origine inconnue. Le deuxième décès fut causé par un syndrome lymphoprolifératif. Les deux enfants transplantés pour hépatite fulminante sont en vie avec une fonction hépatique normale; la récupération neurologique fut complète chez l'un mais l'autre a gardé un état neurologique gravement perturbé (son insuffisance hépatique aiguë fut initialement attribuée au valproate de sodium mais survint vraisemblablement dans le cadre d'un syndrome d'Alpers-Huttenlocher [9] méconnu ("dégénérescence hépatocérébrale»). Vingt-six des 30 greffons originaux $(86 \%)$ sont fonctionnels.

\section{Influence du programme de donneurs vivants sur la mortalité en liste d'attente et l'espérance de survie pour l'ensemble de nos candidats à la greffe}

Ces paramètres ont été étudiés pour tous les patients qui se trouvaient sur notre liste d'attente entre le $1^{\text {er }}$ janvier 1994 et le 31 décembre 1995 (y compris ceux qui avaient été inscrits en 1993 ou ceux qui furent greffés en 1996). Le seul patient transplanté avec donneur vivant en 1993 a été exclu de cette analyse.

\section{- Mortalité en liste d'attente}

Un seul des 30 enfants $(3,3 \%)$ en attente d'une transplantation avec donneur vivant est décédé avant la greffe. Cinq des 49 enfants $(10,2 \%)$ en attente d'un greffon cadavérique sont décédés en liste d'attente. La mortalité globale en liste d'attente a été de $7,6 \%$ (6 sur 79).

\section{- Survie}

La survie calculée à partir de la date de la greffe est de 93,1\% (27 sur 29 enfants) pour le groupe «donneur vivant» et de 79,5\% (35 sur 44 enfants) pour le groupe "greffon cadavérique». Elle est de $84,9 \%$ pour l'ensemble de la série (62 sur 73 enfants).

La survie calculée à partir de la date d'inscription en liste d'attente (qui exprime la véritable espérance de survie des enfants candidats à la greffe) est de $90 \%$ (27 sur 30 enfants) pour le groupe «donneur vivant» et de $71,4 \%$ (35 sur 49 enfants) pour le groupe "greffon cadavérique». Elle est de $78,5 \%$ (62 sur 79 enfants) pour l'ensemble de la série.

\section{Réflexion éthique}

Les choix éthiques à opérer lors du développement d'une nouvelle approche thérapeutique sont guidés par trois principes. L'équilibre risques/bénéfices doit évidemment être favorable. Il convient de le considérer séparément du point de vue du donneur et du receveur puis de façon globale.

Les risques auxquels s'expose le donneur sont liés aux examens radiologiques avec injection de produit de contraste, à l'anesthésie générale et à l'intervention chirurgicale. En transplantation rénale, le risque de complications mortelles d'un don de rein est évalué à 3 sur 10000 donneurs vivants, sur la base de milliers de cas opérés dans le monde [10]. Au début de la transplantation hépatique avec donneur vivant, le risque fut évalué de façon consensuelle à 1 sur 100 , sur la base de la mortalité observée en cas de résection du lobe gauche du foie chez des sujets jeunes dont le foie droit était sain. Depuis la première transplantation avec donneur vivant faite par Raia [11] au Brésil le 8 décembre 1988 (avec décès du receveur au $4^{\mathrm{e}}$ jour postopératoire) et la première transplantation avec survie au long cours faite par Strong [12] en Australie en juillet 1989, 521 observations ont été recensées par le registre mondial des transplantations hépatiques avec donneur vivant [13]. Un décès est survenu par embolie pulmonaire au $4^{\mathrm{e}}$ jour chez une mère obèse, avec tabagisme important et qui n'avait pas arrêté la pilule contraceptive.

Sur la base de l'expérience acquise, le risque de complications mortelles se situe donc actuellement entre 1 sur 500 et 1 sur 1000 . Si le prélèvement du lobe gauche du foie reste une opération majeure, elle est moins complexe, à la lumière de l'expérience, que certains ne le laissent supposer [14], à la condition d'être pratiquée par une équipe très expérimentée en chirurgie du foie. Nous prenons en outre la précaution d'exclure tout donneur présentant le moindre facteur de risque. Le taux de morbidité fut de $14 \%$ chez les 521 donneurs du registre mondial; elle a consisté essentiellement en infections de plaie, fistules biliaires, troubles de l'évacuation gastrique et ulcères gastro-duodénaux.

La résection du lobe gauche emporte $15 \%$ à $20 \%$ de la masse hépatique (environ $35 \%$ quand elle est étendue au segment IV). La capacité de régénération du foie permet une normalisation de la fonction en quelques semaines chez des adultes jeunes en bonne santé. En outre, les donneurs animés d'une forte motivation nous paraissent récupérer plus vite que les patients chirurgicaux habituels et, de ce fait, leur séjour hospitalier postopératoire est bref. Ils sont de plus revus régulièrement et font l'objet d'un suivi psychologique.

Les bénéfices que le donneur vivant retire de sa démarche sont de nature psychologique : faire tout ce qui est en son pouvoir pour contribuer activement à la survie de son enfant et échapper aux affres de l'attente d'un greffon cadavérique qui perturbent gravement les familles (attente de la mort d'un autre, risque du candidat de mourir avant la transplantation, souffrance physique et psychologique du sujet en attente...).

Du point de vue du receveur, les avantages essentiels sont la certitude quasi totale de ne pas décéder en liste d'attente, l'optimalisation de la préparation à la greffe et des suites chirurgicales simplifiées grâce à la 
qualité du greffon. Les risques de complications chirurgicales chez le receveur sont similaires à ceux de la greffe d'un foie partiel ou partagé. Il convient cependant de ne pas banaliser la complexité de l'implantation, chez le receveur, d'un lobe gauche dont les vaisseaux afférents et efférents sont nécessairement courts et de petit calibre. Une longue expérience avec le foie partiel et le foie partagé [15] nous paraît être un prérequis indispensable pour les équipes qui envisagent de débuter un programme de donneur vivant.

Sur le plan psychologique, le risque pour le receveur d'un accident majeur chez le donneur doit être évoqué.

Sur le plan immunologique, une immunosuppression au long cours, probablement à vie selon les connaissances actuelles, est nécessaire tout comme après transplantation d'un greffon cadavérique. Cette immunosuppression comporte des risques, en particulier de développement de néoplasies telles que le syndrome lymphoprolifératif, mais ils sont similaires dans les deux cas de figure.

Aucun bénéfice n'a été observé sur le plan de l'incidence du rejet aigu malgré l'haplo-idendité entre donneur et receveur, à l'exception du groupe de Kyoto [16]. Ce groupe qui a la plus large expérience dans le monde (202 cas en janvier 1996) a observé un taux très bas de rejet aigu $(30 \%)$ qu'aucun autre groupe n'a obtenu jusqu'ici. Le groupe de Chicago [17], qui a été le premier à développer un programme structuré [3], a comparé 38 patients greffés avec donneur vivant et 54 patients transplantés avec un greffon cadavérique de 1990 à 1993. Le taux de rejet aigu fut de $74 \%$ et de $78 \%$ respectivement (différence non significative).

Toutefois, le taux de rejet aigu résistant aux corticoïdes fut significativement réduit $(\mathrm{p}<0,01)$ chez les enfants greffés avec donneur vivant (13\% contre $43 \%$ ). Une tendance à la réduction du taux de rejet chronique fut observée (8\% contre $20 \% ; p<0,1)$ ainsi qu'une perte moins fréquente du greffon par rejet $(3 \%$ contre $9 \%$, différence non significative).

Globalement, l'équilibre des risques et des bénéfices (principe de beneficence des auteurs anglophones) nous parait favorable, tant pour le donneur que pour le receveur. Nous avons recherché l'équilibre le plus favorable dans le protocole initial en limitant le recours au donneur vivant aux enfants en situation clinique stable.

La pénurie d'organes ne permet pas d'offrir à tous les candidats un accès égal à la transplantation en raison de la mortalité en liste d'attente. Le recours au donneur vivant contribue à rétablir une égalité des chances (principe de justice) en réduisant la mortalité préopératoire des enfants en attente d'un greffon cadavérique [18]. En outre, il offre un accès à la transplantation aux enfants qui ne résident pas sur le territoire des organisations régionales d'échange de greffons.

C'est le respect du consentement libre et éclairé et de l'autodétermination du sujet (le donneur vivant) qui pose le plus de problèmes et continue à soulever la controverse [14, 19, 20] . Nous avons pris plusieurs précautions pour respecter ces droits inaliénables. Nous veillons à fournir au donneur une information préalable qui soit claire et la plus complète possible et aborde tous les aspects et risques du prélèvement. Les candidats donneurs sont invités à prendre contact avec d'autres familles qui ont vécu l'expérience.

L'évaluation médicale du donneur est confiée à un médecin interniste qui n'appartient pas à l'équipe de transplantation et dont la mission est de s'assurer de l'absence de tout risque médical. Chaque candidat donneur est longuement rencontré par un psychologue, sous la responsabilité d'un psychiatre, pour vérifier son discernement et le but incontestablement altruiste qui l'anime, selon le prescrit de la loi belge sur le don d'organes ; il convient aussi de dépister les situations à risque de décompensation psychique. Le projet du don est remis en question par l'équipe médicale, pour s'assurer que le candidat donneur agit en toute autonomie, libre de toute pression externe de quelque origine que ce soit (y compris de la part des médecins). La contrainte interne de type moral est inévitable; elle est inhérente à la condition humaine et singulièrement à celle des parents [21]
Nous veillons à respecter la liberté des parents de ne pas retenir l'option du donneur vivant, sans démériter de leur rôle. Le libre choix des parents est rendu possible par l'existence de l'alternative de la transplantation avec greffon cadavérique qui offre une probabilité de survie assez proche des résultats obtenus avec donneur vivant. Ce libre choix est effectivement exercé, y compris dans le sens de l'abstention, comme en attestent neuf parents dont la candidature avait été acceptée mais qui avaient préféré rester en réserve (donneurs de deuxième ligne) au cas où leur enfant se dégraderait dans l'attente d'un greffon cadavérique (leurs enfants ont effectivement bénéficié de cette option).

Pour éviter autant que possible toute forme de pression externe, le protocole initial prévoyait de ne pas parler de l'option «donneur vivant» aux parents qui n'aborderaient pas spontanément la question. En cours d'expérience, nous avons changé de ligne de conduite: lors du premier entretien avec les parents d'un enfant candidat à la greffe, la coordinatrice mentionne l'alternative et invite les parents à reparler de l'option «donneur vivant» s'ils le souhaitent. Certains s'en abstiennent, signalant par ce silence même qu'ils ne la retiennent pas ; dès lors, personne ne leur en reparle plus. Ce changement de ligne de conduite fut dicté par le souci de ménager un égal accès de tous à l'information. Cet amendement a été inclus dans la nouvelle version du protocole, récemment approuvé par la Commission d'Éthique Biomédicale Hospitalo-Facultaire. Un deuxième amendement a été inspiré par l'expérience de trois cas d'hépatite fulminante ou subfulminante où nous avons accepté la candidature spontanée d'un des parents.

Cet amendement porte sur la sélection des candidats qui inclut désormais les enfants en hépatite fulminante, les enfants atteints d'hépatopathie chronique en décompensation et les retransplantations, à la condition que la concertation multidisciplinaire, menée cas par cas, permette de penser que les chances de succès dépassent les risques d'échec (équilibre risques/ 
bénéfices). A terme, nous réévaluerons notre expérience, sans rien concéder en rigueur, dans l'intervalle, en matière de sélection des donneurs.

La transplantation d'organes avec donneur vivant soulève à juste titre d'importantes questions éthiques. Elle doit respecter des principes clairement établis. Il convient cependant de moduler les postulats de départ à la lumière de l'expérience [22]

\section{RÉFÉRENCES}

1. Otte JB, de Ville de Goyet J, Sokal E, Alberti D, Moulin D, de Hemptinne B, Veyckemans F, Van Obbergh L, Carlier M, Clapuyt $\mathrm{Ph}$, Claus D, Jamart J. Size reduction of the donor liver is a safe way to alleviate the shortage of size-matched organs in pediatric liver transplantation. Ann Surg $1990 ; 211: 38-49$.

2. Otte JB, de Ville de Goyet J, Alberti D, Balladur $\mathrm{P}$, de Hemptinne $\mathrm{B}$. The concept and technique of the split liver in clinical transplantation. Surgery $1990 ; 107$ : 605-12.

3. Singer PA, Siegler M, Whitington PF, Lantos JD, Emond JC, Thistlethwaite JR, Broelsch CE. Occasional notes. Ethics of liver transplantation with living donors. NEngl J Med 1989 ; 321 : 620-1.

4. Otte JB, de Ville de Goyet J, Reding R, Hausleithner V, Sokal E, Chardot C, Debande B. Sequential treatment of biliary atresia with Kasaï portoenterostomy and liver transplantation : a review. Hepatology $1994 ; 20$ : 41S-8.

5. Van Obbergh L, Carlier MA, Clement de Clety S, Sokal E, Rennotte MT, Veyckemans F, Dekock M, Frans A, Otte JB. Liver transplantation and pulmonary gas exchange in hypoxaemic children. Am Rev Respir Dis $1993 ; 148$ : 1408-10.
6. Ringe B, Pichlmayr R, Burdelski M. A new technique of hepatic vein reconstruction in partial liver transplantation. Transpl Int $1988 ; 1: 30-5$.

7. Strong R, Ong TH, Pillay P, Wall D, Balderson G, Lynch S. A new method of segmental orthotopic liver transplantation in children. Surgery 1988 ; 104 : 104-7.

8. Emond JC, Heffron TG, Whittington PF, Broelsch CE. Hepatic vein reconstruction in reduced size liver transplantation. Surg Gynecol Obstet 1993 ; 176 : 11-7.

9. Bicknese AR, May W, Hickey WF, Dodson WE. Early childhood hepatocerebral degeneration misdiagnosed as valproate hepatotoxicity. Ann Neurol 1992 ; 92 : 767-75.

10. Najarian JS, Chavers BM, McHugh LE, Matas AJ. 20 years or more of follow-up of living kidney donors. Lancet 1992 ; 340 : 807-10.

11. Raia S, Nery JR, Mies S. Liver transplantation from live donors. Lancet 1989 ; 8861 : 497.

12. Strong RW, Lynch SV, Ong TH. Successful liver transplantation from a living donor to her son. NEngl J Med 1990 ; 322 : 1505-7.

13. Rogiers X, Schroeder T, Tuttlewski K, Broelsch CE. First report of the international living donor liver registry. Barcelona, August 25-30, 1996. The XVIth international congress of the Transplantation Society, abstract, 1996

14. Hartemann E. Transplantation d'organes chez l'enfant. Quels donneurs? A quel prix ? Arch Fr Pediatr 1991 ; 48 : 311-4.

15. De Ville de Goyet J, Hausleithner V, Reding R, Lerut J, Janssen M, Otte JB. Impact of innovative techniques on the waiting list and results in pediatric liver transplantation. Transplantation 1993 ; 56 : 1130-6.

16. Inomata $\mathrm{Y}$, Tanaka $\mathrm{K}$, Egawa $\mathrm{H}$, Uemoto S, Ozaki N, Okajima H, Satomura K, Kiuchi T, Yamaoka Y, Hashida T. The evolution of immunosuppression wit FK 506 in pediatric living-related liver transplantation. Transplantation 1996; 61 : 247-52.
17. Alonso EM, Piper JB, Echols G, Thistlethwaite JR, Whitington PF. Allograft rejection in pediatric recipients of living related liver transplants. Hepatology 1996 ; 23 : 40-3.

18. Emond JC, Leib M. The living-related liver transplant evaluation : linking risk factors and outcome. Liver Transpl Surg 1996; 2 (suppl 1) : 57-63.

19. Whitington PF. Living donor liver transplantation : ethical considerations. J Hepatol $1996 ; 24: 625-7$.

20. Mayer $\mathrm{AD}$. The argument against livedonor liver transplantation. J Hepatol 1996 ; $24: 628-30$.

21. Hennau-Hublet C, Moulin D. Les enjeux éthique et juridique de la transplantation d'organes. Revue mensuelle de l'Université Catholique de Louvain 1996; 71 : 15-21.

22. Moore FD. Three ethical revolutions : ancient assumptions remodeled under pressure of transplantation. Transplant Proc $1988 ; 20: 1061-7$.

\section{J.B. Otte}

Professeur à l'Université catholique de Louvain, chef du service de chirurgie pédiatrique générale et abdominale. Cliniques Universitaires Saint-Luc, avenue Hippocrate 10, 1200 Bruxelles, Belgique.

\section{TIRÉS À PART}

J.B. Otte.

\title{
Institut Curie \\ La Science au Service de l'Homme \\ Remise du Prix Jeanne Loubaresse institut Curie 1995 à Laurent Degos et Anne Dejean-Assémat
}

\begin{abstract}
Le Prix Jeanne Loubaresse - Institut Curie a été remis le mardi 4 février 1997 par le Professeur Jean Bernard, membre de l'Académie des Sciences, à Madame Anne Dejean-Assémat et à Monsieur Laurent Degos lors d'une cérémonie qui s'est déroulée à l'Institut Curie.

Ce prix, d'un montant de $350000 \mathrm{~F}$, a été décerné par le Conseil d'Administration de l'Institut Curie du 15 février 1996, sur proposition d'un jury présidé par Monsieur Roger Monier et composé de Messieurs Philippe Ascher, Thierry Boon-Falleur, Pierre Corvol, Jacques Demaille, Dominique Maraninchi, Bernard Roques, Gérard Toulouse et Gilbert Weill.

Il récompense Monsieur Laurent Degos, Professeur d'Hématologie à l'hôpital Saint-Louis (Université Paris 7) et Madame Anne Dejean-Assémat, Directeur de recherche à l'Inserm (Institut Pasteur), pour avoir montré qu'un dérivé de la vitamine A, l'acide « tout trans rétinoïque », provoque des rémissions complètes dans une forme particulière de leucémie, la leucémie aiguë à promyélocytes, et pour avoir identifié l'anomalie génétique caractéristique de cette leucémie.
\end{abstract}

